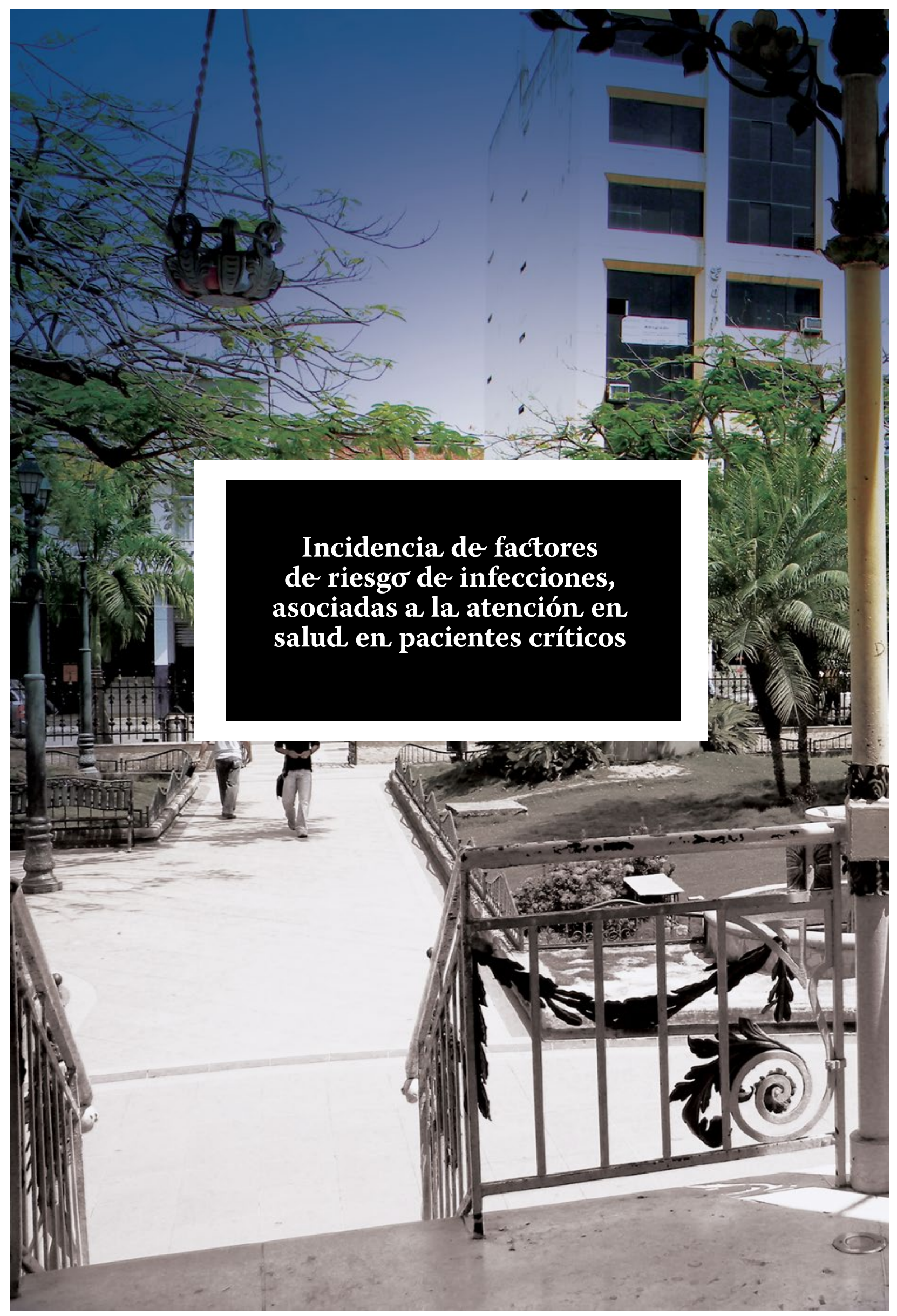




\section{INCIDENCIA DE FACTORES DE RIESGO DE INFECCIONES, ASOCIADAS A LA ATENCIÓN EN SALUD EN PACIENTES CRÍTICOS}

\section{INCIDENCE OF RISK FACTORS IN INFECTIONS ASSOCIATED WITH HEALTH CARE in CRITICAL PATIENTS}

\section{RESUMEN}

Las infecciones asociadas a la atención en salud presentan alto índice en el servicio de Terapia Intensiva por la gravedad del paciente, y el uso de procedimientos invasivos terapéuticos. El estudio se realizó en el Hospital Carlos Andrade Marín de la ciudad de Quito, de forma descriptiva y trasversal, enfocado a 212 profesionales de la salud que fueron observados durante el cuidado directo a los pacientes críticos. Se evaluó la manipulación de tres dispositivos: catéter venoso central, catéter urinario y ventilador mecánico. Resaltando el desequilibrio de medidas asépticas y cumplimiento de protocolos por el personal de salud, con mayor dirección a infección del torrente sanguíneo relacionado con el catéter venoso central. La medición de la adherencia al lavado de manos, según los cinco momentos recomendados por la OMS, demostró con anterioridad que hay deficiencia en: la dotación de insumos y dispositivos médicos, y al momento de realizar procedimientos invasivos y al manejar pacientes con aislamientos respiratorios y de contacto. Sustentando la necesidad de realizar un protocolo de vigilancia que incluya la supervisión de medidas de prevención de higiene de manos, y al realizar procedimientos invasivos para disminuir las tasas de infección asociada a la atención en salud.

PALABRAS CLAVE: Cuidados intensivos; factor de riesgo, infecciones, paciente crítico

Copyright (c) Revista San Gregorio 2019. ISSN 1390-7247; eISSN: 2528-7907 @

\section{ABSTRACT}

Infections associated with health care have high rates in the service of intensive therapy for the severity of the patient, and the use of invasive therapeutic procedures. The study was conducted at the Carlos Andrade Marín Hospital of the Quito city, descriptively and transversely, focused on 212 health professionals who were observed during direct care to critical patients. The manipulation of three devices was assessed: central venous catheter, urinary catheter and mechanical ventilator. Highlighting the imbalance of aseptic measures and compliance with protocols by health personnel, with more direction to infection of the bloodstream related to the central venous catheter. The measurement of adherence to handwashing, according to the five times recommended by WHO, demonstrated previously that there is deficiency in the provision of supplies and medical devices at the time of performing invasive procedures and when handling patients with Respiratory and contact Isolations. Supporting the need to conduct a surveillance protocol that includes monitoring of hand hygiene prevention measures, and in conducting invasive procedures to reduce the rates of infection associated with health care.

KEYWORDS: Risk factor, infections, critical patient, intensive care.

Copyright ( Revista San Gregorio 2019. ISSN 1390-7247; eISSN: 2528-7907 @

\section{Jonathan Alexander González Cano}

Hospital Carlos Andrade Marín. Quito. Pichincha. Ecuador

jonathangonzalezcano@hotmail.es

\section{LILIAM IRIS ESCARIZ BORREgo}




\section{INTRODUCCIÓN}

La carga de infecciones asociadas a la atención en salud (IAAS) constituye la principal esfera de trabajo del programa «Una atención limpia es una atención más segura», direccionada por la Organización Mundial de la Salud (OMS, 2009) donde se han realizado exámenes sistemáticos de la literatura sobre el tema señalando en los países en desarrollo, el riesgo de infección relacionada con la atención sanitaria es de 2 a 20 veces mayor que en los países desarrollados. En algunos países en desarrollo, la proporción de pacientes afectados puede superar el 25\% resaltando la magnitud del problema (Camina. 2018).

Las IAAS son causantes del evento adverso más frecuente durante la prestación de atención sanitaria, y ninguna institución ni país puede afirmar que ha resuelto el problema. Según los datos de varios países entre un 5 y $10 \%$ de los pacientes hospitalizados en Europa y Norteamérica son afectados por las IAAS. En el caso de las regiones de Asia, América Latina y África subsahariana esta cifra sobrepasa el $40 \%$ de los casos de hospitalizaciones (Olaecheaa et al., 2010). En Cuba la vigilancia epidemiológica de las infecciones asociadas a la asistencia sanitaria en los últimos 10 años refleja una tasa global que ha oscilado entre 2,6 y 3,4 por cada 100 egresados, con un promedio anual de 25000 infectados (Arango et al., 2018). El impacto es varias veces superior en los países de ingresos bajos y medianos que en los países de ingresos altos provocando la prolongación de las estancias hospitalarias, discapacidad a largo plazo, una mayor resistencia de los microorganismos a los antimicrobianos, enormes precios adicionales para los sistemas de salud, elevados costos para los pacientes y sus familias, y muertes innecesarias (OMS, 2018).

El impacto negativo de estas sobre el sistema sanitario el que se comporte como indicador para la adopción de estrategias dirigi- das a mitigar esa problemática dentro de los hospitales para el Ministerio de Salud Pública en Ecuador es útil para diseñar estrategias de control y prevención, reducir la morbimortalidad que ocasionan y los gastos institucionales derivados. En esta perspectiva es importante considerar el estudio de factores que están relacionados con las prácticas del personal de salud (Arias, 2018; Salgado, 2017).

El objetivo principal de la presente investigación es determinar los factores de riesgo que inciden en las infecciones asociadas a la atención en salud en pacientes del área de cuidados intensivos del Hospital Carlos Andrade Marín de la ciudad de Quito con el fin de evaluar el estado de los sistemas de control y vigilancia sanitaria. Surgiendo la iniciativa conjunta del Área de Vigilancia Sanitaria y Atención de las Infecciones Asociadas a la Atención en Salud, es significativa la importancia que tienen el fortalecimiento de los Sistemas de Salud de la Organización Panamericana de la Salud en alineación de la Organización Mundial de la Salud.

Es importante el conocimiento de los factores de riesgo de las infecciones asociadas a la atención en salud dentro del territorio ecuatoriano. Esta investigación se enfocó en el área de cuidados intensivos para lograr determinar los principales riesgos en el que está sometido el paciente crítico durante la estancia hospitalaria inherente a la variedad de manipulaciones terapéuticas: invasión del catéter venoso central, colocación de catéter urinario y entubación mecánica, para estabilización y compensación del paciente, donde todo procedimiento invasor realizado por el personal de salud aumenta la propensión a una infección intrahospitalaria (Cohello et al., 2011; Lebeque et al., 2019).

Se considera que en Latinoamérica existe un promedio de $5 \%$ al $12 \%$ de los pacientes internados son poseedores de una infección que no estaba presente al momento de su ingreso hospitalario. Esta temática es endemo-epidémica y con mayor gravedad de acuerdo al nivel de complejidad y más en la unidad de terapia intensiva, donde el paciente permanece invadido para su tratamiento y posible recuperación. Los médicos y el personal de salud tienen noción de que no todas las infecciones hospitalarias son prevenibles, tiene mucha relación los factores de riesgo propios 
del paciente como la patología que lo lleva a la internación y sus comorbilidades añadidas.

Con este estudio se estima que se apliquen medidas rigurosas de prevención lo que logrará reducir las tasas de infecciones asociadas a los cuidados de salud, pero su eliminación a cero es prácticamente imposible.

\section{METODOLOGÍA}

Se realizó un diseño de investigación de tipo cuantitativo, documental bibliográfico, transversal y descriptiva, con el objetivo de identificar los factores de riesgo que inciden en las infecciones asociadas a la atención de salud en pacientes del área de cuidados intensivos del Hospital Carlos Andrade Marín. Enfocado en una población de 212 profesionales de salud concerniente a 110 profesionales de enfermería, 68 médicos y 34 fisioterapistas respiratorios, analizando las actividades específicas de cada profesional que labora de manera rotativa en el ala norte con pacientes clínicos del área de cuidados intensivos, con un tipo de muestra no probabilística, que fueron observadas durante su cuidado directo, aplicando una guía de observación, la cual permitió extraer información para obtener los resultados de la investigación.

Se procederá al cálculo del tamaño de la muestra utilizando la siguiente fórmula (Sares \& Soliz, 2009).

$$
\begin{aligned}
& \mathrm{N} p \mathrm{q} \\
& \mathrm{n}=(\mathrm{N}-1) \mathrm{E} 2+\mathrm{p} \mathrm{q} \\
& \mathrm{z} 2
\end{aligned}
$$

n: tamaño de la muestra.

$\mathrm{N}$ : tamaño de la población es 530 pacientes 0,5

p: posibilidad de que ocurra un evento, $\mathrm{p}=$

q: posibilidad de no ocurrencia de un evento, $q=0,5$

E: error, se considera el 5\%; $E=0,05$

$\mathrm{Z}$ : nivel de confianza, que para el $95 \%, \mathrm{Z}=$ 1,96

\section{Cálculo:}

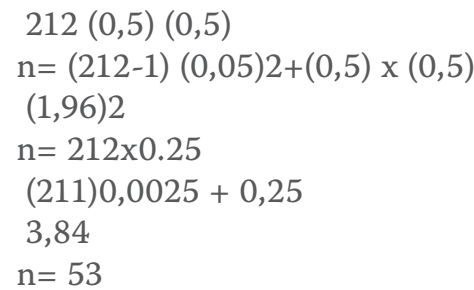

$$
\begin{aligned}
& 0,52+0,25 \\
& 3,84 \\
& \mathrm{n}=53=53=135 . \\
& 0,14+0,25 \quad 0,39 \\
& \text { RESULTADOS }
\end{aligned}
$$

Se expone los resultados obtenidos de la guía de observación aplicado al tamaño de la muestra de 135 profesionales de salud de turnos rotativos entre ellos médicos, enfermeros/as, y fisioterapistas respiratorios que realizan cuidado directo a los pacientes en la unidad de cuidados intensivos. Se analizó la distribución de medidas de barrera adecuada en la manipulación aséptica del catéter venoso central (tabulación cruzada) y se determina que existe una serie de procesos dentro del manejo y cuidado del paciente crítico, donde el dispositivo venoso central está en contacto lineal con el torrente sanguíneo, se logró evidenciar que el $67 \%$ del personal médico realizó una manipulación adecuada, mientras que del grupo de enfermería fue el 55\%, en el acceso central incidió una incorrecta manipulación en los médicos con un 33\% y de los enfermeros/as $27 \%$, omitiendo en reiteradas ocasiones la manera correcta de los procesos de asepsia y protocolos estipulados.

En cuanto a la distribución de aspiración de secreciones en pacientes orointubados (tabulación cruzada), en la observación se midió el manejo de pacientes ventilados con tubo endotraqueal donde interactúan personal de enfermería y terapistas respiratorios, con relación a la aspiración de secreciones en frecuencia durante su turno, mediante los resultados se evidencia que la actividad es ejecutada según la necesidad dada por la condición del paciente crítico siempre y cuando valoren su patrón respiratorio, se evidenció que la mayor frecuencia por turno fue de $71 \%$ y la menor de $29 \%$, también durante el turno se realiza 2 veces la aspiración de secreciones, representando un 61\%, teniendo en cuenta que la condición de los pacientes es sialorreicos e hipersecretores.

En la colocación del catéter urinario (tabulación cruzada) se diferencia entre el personal médico y enfermería al momento de ejecutar el procedimiento, los resultados reflejan que siempre los médicos lo realizan con técnica aséptica el $50 \%$ y, en reiteradas ocasiones por déficit de dispositivos o insumos no lo ejecutan con total técnica estéril en un $49 \%$. Por lo que, en el personal de enfermería, el 54\% 
siempre aplican técnica estéril y el $46 \%$ no lo hacen con toda la complementariedad. Los autores consideran recomendar el abastecimiento de insumos y dispositivos en todos los turnos (mañana, tarde y noche) que garantice el uso de técnica aséptica durante la inserción de catéteres urinarios para prevenir infecciones del tracto urinario.

Con relación a los Momentos del lavado de manos ejecutado por el personal de salud, se aplica en los 5 instantes que enmarca la Organización Mundial de la Salud: Antes de tocar al paciente, previamente al realizar algún procedimiento, después de contacto con fluidos, posteriormente de tocar al paciente y luego del contacto con entorno del mismo; se demuestra que el $20 \%$ de los médicos, el $12 \%$ de enfermeros/as y el $10 \%$ de terapistas respiratorios, aplican los 5 pasos mientras tanto existe el $48 \%$ de no ejecución por parte de los funcionarios. También se identificó la omisión del cuarto momento del lavado de manos por el personal (después de tocar al paciente): un tercio del personal evaluado solo responden a dos intervalos del lavado de manos: médicos $31 \%$, enfermería $36 \%$ y terapistas $38 \%$; otro grupo omite antes y después de tocar al paciente con resultados del: 25\% los médicos, $30 \%$ enfermería y $36 \%$ los terapistas.

Debido a la diversidad de procedimientos se debe disponer de los insumos médicos necesarios en calidad y cantidad para realizar cualquier intervención que el paciente requiera para su estabilización, los resultados indican que: existe un 39\% que refieren siempre hay insumos médicos, mientras que el $50 \%$ respondió que algunas veces no disponen del material necesario para ejecutar el proceso y el 11 \% refirió no disponer de material representando riesgos para la salud.

\section{DISCUSIÓN}

De acuerdo a las bibliografías revisadas, los autores presentan criterios similares relacionados al papel de los factores de riesgo, primordialmente de procedencia invasivas terapéuticas y diagnósticas con el 94\% (Tellez et al., 2009) demostraron que las neumonías nosocomiales asociadas a ventilación mecánica aparecen precozmente (37.6\%) así como, las infecciones del torrente sanguíneo asociadas a catéter venoso central manifiestan una tendencia a incrementarse (23.9\%).
El uso de catéteres y terapia intravenosa constituyen el principal factor de riesgo para el desarrollo de bacteriemias primarias. La bacteriemia es, junto con la neumonía asociada a la ventilación mecánica, la infección nosocomial más frecuente en los pacientes críticos y se asocia a una importante morbimortalidad y a la contaminación de soluciones en las áreas de pediatría y unidades de cuidados intensivos neonatal. La mezcla de soluciones y la falta de capacitación del personal incrementan el riesgo de dichas infecciones (Secretaria de Salud México, 2011)

Mediante el análisis de la bibliografía se considera frecuente el uso de catéteres venosos centrales en el paciente crítico que ocasiona el paso directo de microorganismos a la circulación sistémica, como la bacteriemia relacionada con catéter, seguido de la infección nosocomial (IN) en frecuencia en 10,07\% de todas las IN, con una incidencia de 1,42 BRC por 1.000 días de uso de catéter (Tiller et al., 2018; López \& Cortés. 2012).

Se demostró en el estudio de (Vieira et al., 2013) sobre el catéter central que es realizada de forma aséptica en mayor porcentaje por enfermería y con un margen del $6 \%$ por los médicos, pero existe un porcentaje del $60 \%$ de incorrecta manipulación aséptica del catéter venoso central. Para los pacientes en la unidad de cuidados intensivos (UCI), su disminución se basa principalmente en medidas preventivas simples.

Se realiza la aspiración de secreciones a los pacientes de la unidad de cuidados intensivos la cual se ejecuta mediante previa valoración del patrón respiratorio y marcado por la estabilidad del paciente ventilado, en muchas ocasiones se realiza según la necesidad del mismo, con una frecuencia mayor de 2-3 aspiraciones por turno, siempre realizada por personal de enfermería o terapista respiratorio, al justificar que hay pacientes hipersecretores, variando de acuerdo a la condición del paciente crítico.

Según Ponce de León et al. (1999) en su estudio sobre la determinación de la frecuencia de infecciones nosocomiales en el Instituto Nacional de la Nutrición Salvador Zubirán (INNSZ) de México, la infección más frecuente fueron las vías urinarias con un $26.6 \%$ del total de episodios, realizando una comparación de los mismos entre los años 1991-1993 
y 1996, se observó una disminución significativa.

La colocación del catéter urinario de acuerdo al protocolo de la unidad de cuidados intensivos del hospital, fue realizado por el personal médico siempre, con técnica y material aséptico $50 \%$ y a veces $49 \%$, a comparación del personal de enfermería siempre con técnica y material estéril $54 \%$ y en ocasiones a veces en $46 \%$, basado en el desequilibrio de dotación de insumos y dispositivos médicos, omisión de los cinco momentos y tiempo de duración del lavado de manos, este quebranto se localizó con mayor frecuencia en turnos de la tarde y noche, donde el sistema de vigilancia no está totalmente activo (Rodríguez, 2016; Ruiz et al., 2014).

Según estudios del Hospital Clínico-quirúrgico Docente "Saturnino Lora Torres" de Cuba, el mantenimiento del catéter permanente la higiene del perineo (Barriga et al., 2014) se ha revelado como una estrategia eficaz en la disminución de la incidencia de ITU, la cual necesita ser practicada diariamente en el meato uretral, con agua y jabón durante el baño. En esta investigación se utilizó el sistema colector abierto en $67,6 \%$ de los afectados, por tanto, el riesgo de infección es de aproximadamente de $5 \%$ por día de cateterización.

Otro estudio del Centro Colaborador de la OMS para el Desarrollo de la Investigación en Enfermería de Brasil, para los autores (Mazzo et al., 2015) la duración de la sonda es un factor determinante en la probabilidad de adquirir una ITU.

En el estudio realizado en esta investigación la gran mayoría del personal ejecuta la higiene de manos según las disposiciones de la Organización Mundial de la Salud con los grupos de médicos y enfermería mayor al $50 \%$ y con los terapistas respiratorios un $45 \%$, teniendo la mitad del porcentaje sin aplicar durante su turno con un tiempo de duración 40 a 60 segundos y un valor mayor al $40 \%$ tal como se encuentra estipulado en el protocolo, y con un margen de $22 \%$ en personas que realizan el lavado en menos de veinte segundos, no cumpliendo con la eliminación de microorganismos que se encuentran en las manos del personal (Rivera et al., 2015).

La Alianza Mundial para la Seguridad del Paciente inició con el eslogan "La cirugía se- gura salva vidas" siendo una de la fortaleza de la Organización Mundial de la Salud (OMS) con la convicción de reducir en todo el mundo el número de muertes de origen quirúrgico y las de hospitalización. Según (Secretaría de Salud, 2011) enfatiza la correcta higiene de manos en la intervención de infecciones intrahospitalarias, a pesar de la importancia que tiene este elemento básico, en la atención clínica se demostró que el 85 \% de los hospitales participan en las campañas de la OMS, pero únicamente el 58\% cuentan con evidencia de su adhesión a la aplicación de la higiene de manos.

Se muestran evidencia científica y médica que el personal de salud debe cumplir con la higiene de manos, en España, sólo un 40\% de los profesionales afirma correctamente que tras el lavado de las manos con agua y jabón no es necesario realizar fricción con preparados de base alcohólica. (Fonseca et al., 2014). Solo el $42 \%$ de todo el personal aplicar el lavado de manos en los cinco momentos con el paciente.

Se confirma que las infecciones asociadas a la salud son un problema de salud pública en los hospitales, pues significa un alto costo socioeconómico y ocasiona efectos colaterales como la disminución de posibilidades en el uso de camas para pacientes con otras patologías; por lo que la vigilancia epidemiológica es necesaria y fundamental, en la prevención y control de las infecciones y de disminuir la morbilidad y mortalidad, así como la reducción de costo (Alba-Leonel et al., 2014; Hernández et al., 2001).

\section{CONCLUSIONES}

La investigación sobre la Incidencia de factores de riesgo en infecciones asociadas a la atención en salud en pacientes del área de cuidados intensivos, Hospital Carlos Andrade Marín, Quito 2018 concluye, que los objetivos que se plantearon al inicio se han alcanzado y se confirmaron verificando a continuación lo siguiente:

Los factores de riesgo que inciden en las infecciones asociadas a la atención en salud se pueden disminuir, realizando una vigilancia activa diaria de tres dispositivos: catéter venoso central, catéter urinario y el ventilador mecánico en pacientes del área de cuidados intensivos a cargo del personal de salud: mé- 
dicos, enfermeras/os y fisioterapistas respiratorios del Hospital Carlos Andrade Marín.

Se demostró la relación de los procesos de atención referente a la manipulación de los dispositivos empleados por el personal, con la línea de prevención de infecciones asociadas a la atención en salud en pacientes del área de cuidados intensivos; se evidenció una prevalencia de $73 \%$ referente a la incorrecta manipulación del catéter venoso central.

Se estableció la técnica de lavado de manos, marcando un procedimiento básico e indispensable con ejecución de la mitad del 100\% a través del personal de salud, pero existe un desequilibrio evidenciado y controlado mediante los momentos del lavado de manos.

Se analizó la incidencia del manejo y disponibilidad de los insumos médicos al aplicar los procedimientos durante el proceso de atención en salud, no disponibilidad del 100\% de equipos, dispositivos e insumos; se aclara la cobertura que existe en horario de la mañana, pero en turnos de la tarde y noche no se logra cubrir en reiteradas ocasiones, llevando un proceso incompleto con la invasión diagnóstica y terapéutica del paciente crítico.

\section{REFERENCIAS BIBLIOGRÁFICAS}

Alba-Leonel, A., Fajardo-Ortiz, G., \& PapaquiHernández, J. (2014). La importancia del lavado de manos por parte del personal a cargo del cuidado de los pacientes hospitalizados. Revista de enfermería neurológica, 13(1), 19-24.

Arango Díaz, A. et al (2018). Epidemiología de las infecciones asociadas a la asistencia sanitaria. Acta Médica del Centro, 12(3), 262-272.

Arias, M. (2018). El colombiano. Falta de insumos médicos agrava crisis hospitalaria: Obtenido fecha de marzo 2018. https://www.elcolombiano.com/antioquia/ congestion-en-urgencias-de-hospitales-de-tercer-nivelen-medellin-NC10123907

Barriga, J, Cerda, J, Abarca, K, Ferrés, M, Fajuri, P, Riquelme, M, Carrillo, D, \& Clavería, C. (2014). Infecciones asociadas a la atención en salud (IAAS) en pacientes pediátricos post-operados de cardiopatías congénitas. Revista chilena de infectología, 31(1), 16-20. https://dx.doi.org/10.4067/S0716-10182014000100002

Camina, M. L., Perdomini, M., Picca, Y., \& Pino, T. El cuidado. enfermería - Florence Nghtingale. Obtenido fecha agosto de 2018 de http://teoriasdeenfermeriauns. blogspot.com/2012/06/florence-nightingale.html

Coelho, M.S., Silva Arruda, C., \& Faria Simões, S.M. (2011). Higiene de manos como estrategia fundamental en el control de infección hospitalaria: un estudio cuantitativo. Enfermería Global, 10(21).

Comisión de las Comunidades Europeas. (2008). Comisión de las Comunidades Europeas., (págs. 56-60). Bruselas. Obtenido el 24 de Julio de 2018, de http:// ec.europa.eu/health/ph_systems/docs/patient com 2008

Complejo Hospitalario Universitario de Albacete. (2014). Área de Enfermería. Protocolos de Enfermería-Unidad de Cuidados Intensivos. Obtenido diciembre de 2018 de http://www.chospab.es/enfermeria/protocolos/uci/ neumonia_prevencion.htm

El Universo. (24 de Julio de 2014). Insumos médicos faltan en IESS, dicen pacientes. Falta de insumos médicos, págs. 10-15. Obtenido de https://www.eluniverso.com/ noticias $/ 2014 / 07 / 24 /$ nota/3272826/insumos-medicosfaltan-iess-dicen-pacientes
Fonseca-Ruíz, N., \& Restrepo, S., \& Pérez, N., \& Molina, F., \& Ortíz, G., \& (2014). Infecciones asociadas a dispositivos en unidades de cuidado intensivo académicas vs no académicas. ¿Hay diferencia? CES Medicina, 28 (2), 221-232.

Hernández P, Glenn, Dalmazzo A, Roberto, Gabriela De la Cerda, S, Saavedra M, Carmen, \& Calvo A, Mario. (2001). Prevención de la neumonía asociada a ventilación mecánica. Revista chilena de infectología, 18 (Supl. 2), 66-76. $\quad$ https://dx.doi.org/10.4067/S071610182001018200004

Lebeque Pérez, Yamila, Morris Quevedo, Humberto J., \& Calás Viamonte, Nerys. (2006). Infecciones nosocomiales: incidencia de la Pseudomonas aeruginosa. Revista Cubana de Medicina, 45(1) Recuperado en 04 de junio de 2018, de http://scielo.sld.cu/scielo.php?script=sci arttext\&pid=S0034-75232006000100005\&lng=es\&tlng= es.

López, J., \& Cortés, J. (2012). Colonización e infección de la vía urinaria en el paciente críticamente enfermo. Medicina intensiva, 36(2), 143-151.

Mazzo, A., Bardivia, C. B., Jorge, B. M., Júnior, V. D. S., Fumincelli, L., \& Mendes, I. A. C. (2015). Cateterismo urinario permanente: práctica clínica. Enfermería Global, 14.(38), 50-59.

Ministerio de Salud de Colombia. dataiFX.com. Obtenido 24 de Julio de 2018 de http://www.dataifx.com/noticias/ colombia-gasta\%C2\%A0700-millones-

en\%C2\%A0pacientes-con-infecciones-recibidasen\%C2\%A0atenci\%C3\%B3n-hospitalaria

Ministerio de Salud Pública. (2010). Por un parto culturalmente adecuado: un derecho de las mujeres y de los recién nacidos. Quito: Care.

Ministerio de Salud Pública. (2011). Guía metodológica para la atención de la salud materna culturalmente adecuada. Atención del Embarazo, Parto y Post parto culturalmente adecuados (págs. 1-30). Ecuador.

Olaecheaa, J. Insaustib, A. Blancoc \& Luqued. P (2010). Epidemiologia e impacto de las infecciones nosocomiales. Medicina Intensiva. 34(4):256-267 
Organización Mundial de la Salud. (2009). La OMS y su iniciativa Alianza Mundial para la Seguridad del Paciente. Argentina: Archivos Argentinos de Pediatría. 107(5):385-386

Organización Mundial de la Salud. (2018). Carga mundial de infecciones asociadas a la atención sanitaria. (c) 2018 WHO. Recuperado el 24 de Julio de 2018, de http://www. who.int/gpsc/country_work/burden_hcai/es/

Ponce de León, S., Rangel-Frausto, S., Elías-López, J. I., Romero-Oliveros, C., \& Huertas-Jiménez, M. (1999). Infecciones nosocomiales: tendencias seculares de un programa de control en México. salud pública de México, 41, S05-S11.

Rivera, M. (21 de junio de 2017). 10 \% del dinero para insumos médicos se pierde en corrupción. Recuperado de El Espectador: https://www.elespectador.com/ noticias/bogota/10-del-dinero-para-insumos-medicosse-pierde-en-corrupcion-articulo-699265

Rodríguez, F. (27 de Julio de 2016). Prevención y Control de la Infección Nosocomial. Recuperado de https://www. franrzmn.com/prevencion-y-control-de-la-infeccionnosocomial/

Ruiz, N. J. F., Mesa, S. L. R., Pérez, N., Molina, J. F., \& San Juan, G. O. (2014). Infecciones asociadas a dispositivos en unidades de cuidado intensivo académicas vs no académicas. ¿Hay diferencia? Revista CES Medicina, 28(2), 221-232.

Salgado, E. (2017). Ecuador con alta tasa de mortalidad por infecciones nosocomiales. Redacción Médica - Salud Pública Ecuador, 1-10. Recuperado el 30 de Julio de 2018, de https://www.redaccionmedica.ec/secciones/saludpublica/ecuador-con-la-mayor-tasa-de-mortalidad-porinfecciones-nosocomiales-89901

Sares Carrión, V. \& Soliz Sánchez, J. (2009). Cumplimiento del lavado de manos por parte del personal de salud del servicio de emergencia del Hospital José Carrasco Arteaga, Cuenca-Ecuador (Bachelor's thesis).

Secretaría de Salud. (2011). Medición de la prevalencia de infecciones nosocomiales en hospitales generales de las principales instituciones públicas de salud. México, D.F.: Secretaría de Salud.

Tellez Velásquez, R., Sarduy Ramos, C. M., Rodríguez Pérez, J., Rodríguez Acosta, R., \& Segura Pujal, L. (2008). Infecciones intrahospitalarias en los servicios clínicos. Revista Archivo Médico de Camagüey, 12(2), 0-0.

Tiller García. J, Aranda Aya A \& Morales E. (2018). Infecciones asociadas a dispositivos intravasculares en las Unidades de Cuidados Intensivos Adultos. Tesis de Especialización de la Universidad Santo Tomás de Aquino. Bogotá

Vieira, K., Nascimento, C. E., de Andrade, P. S., Enders, B. C., Coura, A. S., \& Dutra, M. O. M. (2014). Acciones de enfermería para la prevención de la neumonía asociada a ventilación mecánica: revisión sistemática. Enfermería Global, 13(35), 338-349.

Villanueva-Meyer, M. (2018). Ignaz Semmel Weis (18181865): Padre del control de las infecciones. Galenus Revista para los médicos de Puerto Rico, 20-30. http:// www.galenusrevista.com/?Ignaz-Semmelweis-18181865-Padre 